\title{
Raucherbann tut dem Herzen gut
}

\begin{abstract}
Bereits ein Jahr nach dem generellen Rauchverbot in öffentlichen geschlossenen Räumen macht sich diese Maßnahme in England in einem signifikanten Rückgang der Krankenhausaufnahmerate wegen akutem Myokardinfarkt bemerkbar.
\end{abstract}

- In zahlreichen Studien und Metaanalysen konnte ein erhöhtes koronares Risiko durch Passivrauchen nachgewiesen werden. Umgekehrt gibt es aus mittlerweile mehreren Ländern Beobachtungsdaten, wonach nach Einführung eines Rauchverbots in öffentlichen geschlossenen Räumen die Rate von Myokardinfarkten zurückgegangen ist. Nur zwei Studien aus Neuseeland und Piemont konnten diesen Zusammenhang nicht beobachten.

Nun wird aus England auf der Basis einer Gesamtbevölkerungszahl von 49 Mio. und den relativ validen Daten des nationalen Gesundheitsdienstes der Effekt des allgemeinen Rauchverbots seit dem 1. Juli 2007 im Jahr nach dieser Maßnahme analysiert. Dabei wurde besonderer Wert auf die Korrektur der

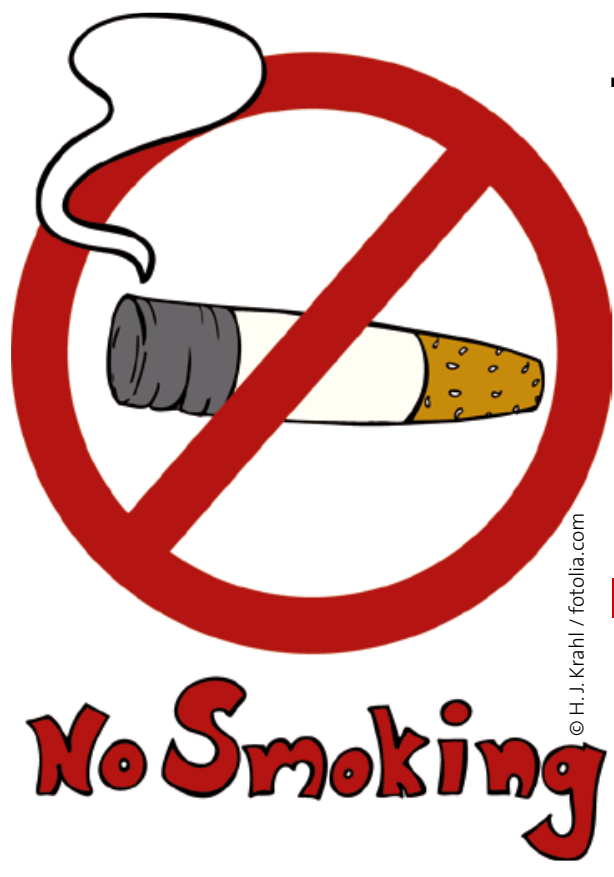

Daten für säkulare und saisonale Trends und weitere konfrontierende Faktoren gelegt.

Im Jahr nach Inkrafttreten des allgemeinen Rauchverbots kam es zu einem geringen, aber statistisch signifikanten Rückgang der Akutaufnahme wegen Myokardinfarkt um 2,4\%. Der Rückgang betraf Männer und Frauen über 60 Jahre in gleicher Weise und war auch bei Männern unter 60, jedoch nicht bei jüngeren Frauen zu beobachten.
- M. Sims et al.

(Korr.: Anna Gilmore, School for Health, University of Bath, Bath BA2 7AY, e-mail: a.gilmore@bath.ac.uk): Short term impact of smoke-free legislation in England: retrospective analysis of hospital admissions for myocardial infarction. Brit. Med. J. 340 (2010) 7760, c2161

\section{Kommentar}

Das Ergebnis mag im Vergleich zu den Daten aus anderen Ländern als gering erscheinen, doch darf man nicht vergessen, dass gerade in England bereits vor der gesetzlichen Einführung des Rauchverbots in öffentlichen Gebäuden, am Arbeitsplatz und in Lokalen viele Institutionen von ihren Beschäftigten bzw. Kunden verlangt haben, auf das Rauchen zu verzichten. Je geringer die Exposition gegenüber Zigarettenrauch vor einer gesetzlichen Maßnahme war, desto weniger ausgeprägt ist der Effekt eines gesetzlichen Rauchverbots. Die vorliegende Studie besticht auch durch einen hohen Grad an Kontrolle gegenüber konfrontierenden Faktoren, was die Aussage vielleicht weniger spektakulär, aber umso valider macht.

H. S. FÜEßL =

\section{Hilft Akupunktur beim Nikotinentzug?}

Beim Nikotinentzug soll Akupunktur Wunder wirken - wird oft behauptet. Wissenschaftlichen Studien hält diese Behauptung nicht stand.

- Koreanische Forscher haben 80 Raucher, die die Zigaretten aufgeben wollten, in zwei Gruppen randomisiert. Die Verumpatienten erhielten zwei Wochen lang regelmäßig Akupunktur, während die Kontrollgruppe mit Scheinakupunktur behandelt wurde. Diese bestand aus Nadelung an Nichtakupunkturpunkten.

Als primärer Studienendpunkt war die Intensität der Entzugssymptome definiert wurden. Die Auswertung zeigte, dass im Verlauf der zweiwöchigen Therapie sowie bei einer Nachbeobachtung nach vier Wochen die Entzugssymptome in beiden Gruppen ähnlich rückläufig waren und keine Gruppendifferenzen aufwiesen.

\section{Kommentar}

Die Akupunktur wird weltweit zum Nikotinentzug empfohlen. Zahlreiche Voruntersuchungen sowie ein CochraneReview zeigen, dass diese Vorgehensweise nicht wirklich effektiv ist. Die hier vorgelegte, gut gemachte Studie belegt diesen
Befund abermals. Somit ist es nunmehr an der Zeit, glaube ich, dass wir unseren Patienten reinen Wein einschenken und sagen, dass es sich hier nicht um ein Verfahren handelt, von dem mehr als Placeboeffekte zu erwarten sind.

E. ERNST -

\footnotetext{
- M. K. Hyun et al.

Body acupuncture for nicotine withdrawal symptoms: A randomized placebo-controlled trial. Evid. Based Complement. Alternat. Med. 7 (2010) $233-238$
} 